\title{
Paciente acometido por Angina de Ludwig com grave progressão reabilitado com próteses dentárias: relato de caso
}

Pacient affected by Ludwig's Angina with serious progression rehabilitated with dental prosthetics: case report

Paciente acometido por Angina de Ludwig con grave progresión rehabilitado con prótesis dentales: reporte de caso

\author{
Adriana Caroline LEITE ${ }^{1}$ \\ Daniele Maria dos Santos GOES 1 \\ Ricardo SHIBAYAMA2 \\ Glaykon Alex Vitti STABILE 3 \\ Marcelo Medeiros BATTISTETTI ${ }^{3}$ \\ Cecília Luiz Pereira STABILE ${ }^{3}$
}

${ }^{I}$ Graduanda, Curso de Odontologia, Universidade Estadual de Londrina (UEL) 86057-970 Londrina - PR, Brasil

${ }^{2}$ Área de Prótese Dental, Departamento de Odontologia Restauradora, Universidade Estadual de Londrina (UEL) 86057-970 Londrina-PR, Brasil

${ }^{3}$ Área de Cirurgia Bucal, Departamento de Medicina Oral e Odontologia Infantil, Universidade Estadual de Londrina (UEL) 86057-970 Londrina-PR, Brasil

\begin{abstract}
Resumo
O objetivo deste trabalho é relatar e discutir um caso clínico de um paciente acometido por Angina de Ludwig que evoluiu gravemente para mediastinite. O paciente compareceu inicialmente ao Pronto Socorro do Hospital Universitário da Universidade Estadual de Londrina e após diagnóstico de Angina de Ludwig foi internado e tratado de forma emergencial. O tratamento foi multiprofisssional e constituiu-se em abordagem cirúrgica para descompressão dos tecidos, administração de antimicrobianos e remoção da causa da infecção. Mesmo após a primeira abordagem cirúrgica, o paciente evoluiu gravemente e houve a necessidade de reabordagem da região cervical e torácica. $\mathrm{O}$ paciente necessitou de um mês de internação hospitalar até que o quadro infeccioso fosse estabilizado. Após o incidente, o paciente se apresentava com baixa autoestima e com sua relação interpessoal afetada. Desta forma, foi encaminhado para a Clínica Odontológica Universitária da Universidade Estadual de Londrina onde foi reabilitado com prótese total superior e prótese parcial removível inferior a fim de devolver grande parte da função mastigatória, estética e, consequentemente, qualidade de vida.
\end{abstract}

Descritores: Angina de Ludwig; Infecção; Prótese Dentária; Saúde Bucal.

\section{Abstract}

The purpose of this study is to report and discuss a case of a patiente affected by Ludwig's angina that progressed severely to mediastinitis. The patient attended the Emergency Room at the University Hospital, State University of Londrina, and was admitted and treated in an emergencial manner. The treatment was multiprofessional and consisted in a surgical approach for tissue descompression, antibiotic administration and removal of the infection origin. Even after the first surgical approach, his clinical condition deteriorated and a new surgical intervention was necessary. The patient needed a 30-day hospital stay until the infection stabilization. After the incident, the patient had low self-esteem and his interpersonal relatioships were affected. Therefore, he was referred to the University Dental Clinic of the State University of Londrina where he was rehabilitated with an upper total prosthesis and a lower removable partial prosthesis. Treatment was able to recover masticatory and aesthetic funtion, and consequently, life quality.

Descriptors: Ludwig's Angina; Infection; Dental Prosthesis; Oral Health.

\section{Resumen}

El objetivo de este trabajo es relatar y discutir un caso clínico de un paciente acometido por Angina de Ludwig que evolucionó gravemente a una mediastinitis. El paciente asistió al Pronto Socorro del Hospital Universitario de la Universidad Estatal de Londrina y tras el diagnóstico de Angina de Ludwig fue internado y tratado emergencialmente. El tratamiento fue multiprofesional y se constituyó en un abordaje quirúrgico para la descompresión de los tejidos, administración de antimicrobianos y eliminación de la causa de la infección. Incluso después del primer abordaje quirúrgico, el paciente evolucionó gravemente y hubo la necesidad de nueva abordaje de la región cervical y torácica. El paciente necesitó de un mes de internación hospitalaria hasta que el cuadro infeccioso se estabilizara. Después del incidente, el paciente se presentaba con baja autoestima y con sus relaciones interpersonales afectadas. De esta forma, fue encaminado a la Clínica Odontológica Universitaria de la Universidad Estadual de Londrina donde fue rehabilitado con prótesis total superior y prótesis parcial removible inferior a fin de devolver gran parte de la función masticatoria, estética y, consecuentemente, calidad de vida.

Descriptores: Angina de Ludwig; Infección; Prótesis Dental; Salud Bucal.

\section{INTRODUÇÃO}

Apesar da melhora da condição oral da população de forma geral, ainda existem casos de infecções odontológicas que se agravam a ponto de colocar a vida do paciente em risco. Uma das complicações causadas por infecções odontogênicas é a Angina de Ludwig (AL), caracterizada pelo envolvimento dos espaços sublingual, submentoniano e submandibular. A Angina de Ludwig foi descrita pela primeira vez em 1856 pelo médico alemão Wilhelm Friedrich Von Ludwig como uma doença infecciosa grave que acomete rapidamente o assoalho bucal em forma de celulite ${ }^{1 / 2}$.

A etiologia da Angina de Ludwig é na maioria das vezes odontológica, principalmente advinda de molares inferiores por causarem infecção que se espalha mais facilmente para os espaços submandibulares. Quando de origem não odontogênica pode ser advinda de infecções tonsilofaríngeas, sialolitíases, neoplasias e fraturas mandibulares ${ }^{3}$.

$\mathrm{Na}$ maioria dos casos, a infecção é polimicrobiana e sua propagação é facilitada pelo sinergismo de microrganismos aeróbicos e anaeróbicos, pertencentes à flora microbiana da mucosa orofaríngea. As bactérias anaeróbicas mais comumente encontradas são Streptococcus viridans, Streptococcus pyogenes, Staphylococcus aureus, Bhaemolytic e Klebsiella pneumoniae, enquanto as anaeróbicas são Prevotella, Fusobacterium e Peptostreptococcus ${ }^{4,5}$.

Algumas complicações ameaçadoras à vida estão associadas à Angina de Ludwig, como obstrução das vias aéreas, infecção das vias aéreas, pneumonia, empiema pleural, mediastinite, pericardite, fasciíte necrosante cervico-facial, trombose da veia jugular, coagulação intravascular 
disseminada, trombose do seio dural e sepse. Sendo assim, a intervenção deve ser feita o mais rápido possível para evitar a disseminação e agravamento da infecção ${ }^{6,7}$.

O tratamento da Angina de Ludwig consiste na manutenção e proteção das vias aéreas através de observação e monitorização do paciente, entubação orotraqueal, entubação naso-traqueal ou traqueostomia, exploração cirúrgica para permitir a drenagem da coleção purulenta e descompressão dos tecidos, administração adequada de antibacterianos, suporte do estado imunológico quando necessário e remoção do agente causador ${ }^{8}$.

O paciente acometido por uma condição grave como a Angina de Ludwig comumente apresenta-se como um paciente negligente em relação a saúde bucal, podendo, ou não, apresentar agravamento sistêmico. Frequentemente, o paciente apresenta um ou mais elementos dentários em condições precárias e que precisam ser extraídos. Por isso, além de tratar a infecção, convencer o paciente de que o quadro se instalou devido a um descuido com a saúde oral é fundamental. Deve-se motivar o indivíduo e mostrar que é necessário uma mudança de atitude ${ }^{9}$.

A condição bucal influencia diretamente na qualidade de vida das pessoas tanto no nível biológico (mastigação, fonação e função articular) quanto no psicológico e social (autoestima, comunicação e estética). Dentes destruídos e perdas dentárias são comumente encontrados em pacientes acometidos por infecções graves, e a reabilitação oral deste paciente é um desafio para o cirurgião dentista $^{10}$.

Após a estabilização do quadro infeccioso, as próteses dentárias são alternativas para reconstituir a autoestima e imagem destes pacientes frente a sociedade. A condição financeira de cada paciente é um fator determinante para a escolha do tipo de prótese a ser instalada. A decisão deve ser feita pelo cirurgião dentista em conjunto com o paciente, levando em consideração as indicações e conveniências para cada caso ${ }^{11,12}$.

O objetivo deste trabalho foi realizar uma revisão de literatura sobre a Angina de Ludwig, além de apresentar e discutir um caso em que o paciente compareceu ao Hospital Universitário da Universidade Estadual de Londrina (UEL) diagnosticado com Angina de Ludwig que evoluiu para mediastinite, fazendo com que o paciente transcorresse um mês de internação hospitalar. Após o incidente, o paciente se encontrava sem autoestima e com suas relações sociais afetadas, o que levou a procurar atendimento na Clínica Odontológica da UEL. O paciente foi orientado quanto a sua saúde bucal e foram confeccionadas próteses dentárias a fim de devolver parte da função mastigatória, estética $\mathrm{e}$, consequentemente, qualidade de vida.

\section{CASO CLÍNICO}

Paciente do sexo masculino, 42 anos, leucoderma, tabagista e etilista, compareceu ao Pronto Socorro do Hospital Universitário de Londrina queixando-se de edema na face acompanhado de dor intensa. Durante a anamnese referiu dor na região do elemento 37 há 5 dias, evoluindo com febre, trismo, aumento de volume em face e episódio de drenagem espontânea intrabucal. Ao exame físico observou-se aumento de volume e hiperemia na face, região cervical e tórax com sinal de cacifo positivo, acompanhado de disfagia, dispneia e trismo. No exame físico intrabucal observou-se péssima condição de higiene oral, vários elementos dentários fraturados e/ou cariados e um aumento de volume na região sublingual (Figura 1). Foi administrado inicialmente, pela equipe médica da Emergência, Penicilina Benzatina IM 120.000 UI e Metronidazol 500mg EV, Tramadol 100mg, Plasil $10 \mathrm{mg}$, Morfina $4 \mathrm{mg}$ e Hidrocortisona $500 \mathrm{mg}$. Visto que a infecção era de origem odontogênica, a equipe da residência de Cirurgia e Traumatologia Bucomaxilo-facial (CTBMF) foi acionada. Foi requisitada tomografia computadorizada que mostrou aumento de volume nos espaços fasciais e desvio de via aérea (Figura 2).

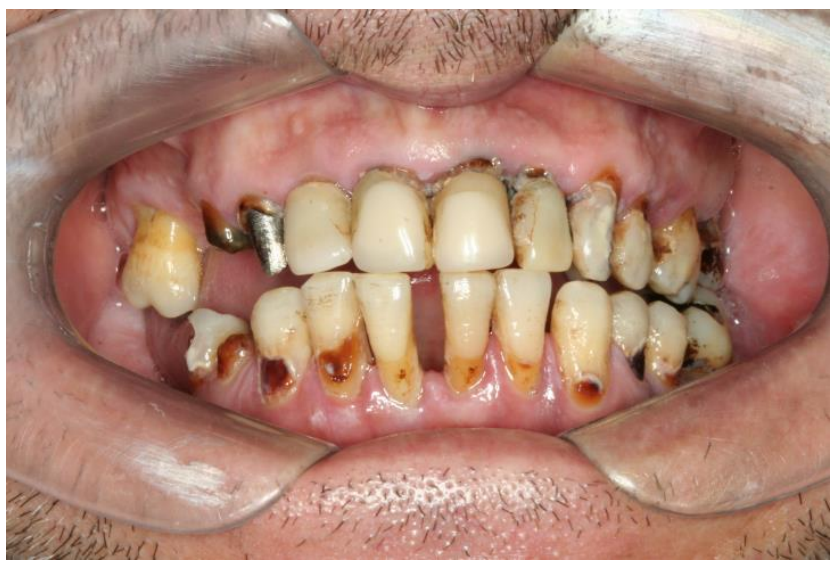

Figura 1: Aspecto intrabucal do paciente mostrando dentes em situação precária.

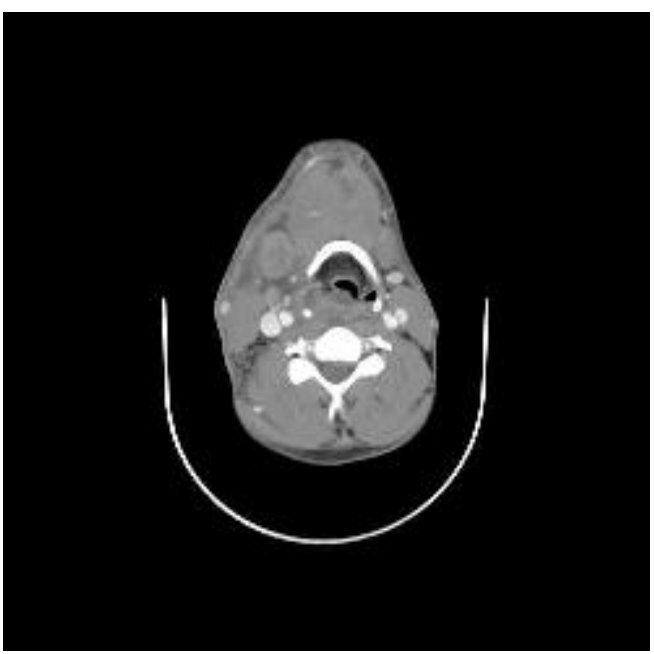

Figura 2: Corte axial de TC de cabeça e pescoço mostrando aumento de volume na região submandibular e desvio da via área. 
O paciente foi submetido a procedimento cirúrgico para drenagem e descompressão dos tecidos de forma multidisciplinar. Foi feito, pelo serviço de CTBMF, acesso transcervical bilateral, exodontias múltiplas, colocação de 3 drenos Penrose e 1 semirrígido (Figura 3). Além disso, a equipe de cirurgia torácica realizou toracotomia, mediastinostomia, traqueostomia e drenagem torácica bilateral, encontrando necrose extensa de musculatura pré-traqueal, com saída de secreção purulenta em grande quantidade; necrose de platisma, músculos hioideos e de fáscia cervical profunda. A antibioticoterapia consistiu na administração de Vancomicina e Tazocin, porém, houve troca da medicação por 4 vezes durante a internação.

$\mathrm{O}$ paciente evoluiu mal, com piora do quadro clínico no dia seguinte à primeira cirurgia exploradora, ficando em estado gravíssimo, com necessidade de reabordagem cirúrgica. Após a reabordagem cirúrgica e recolocação dos drenos, o paciente ainda permaneceu 10 dias em UTI até estabilização do quadro infeccioso. Além disso, o paciente desenvolveu úlcera sacral sendo necessário debridamento da ferida, o que retardou a sua alta hospitalar, a qual se deu 30 dias após a internação. $\mathrm{O}$ paciente continuou em acompanhamento ambulatorial pelo serviço de residência em CTBMF, que executou mais algumas exodontias após estabilização da saúde geral (Figura 4).

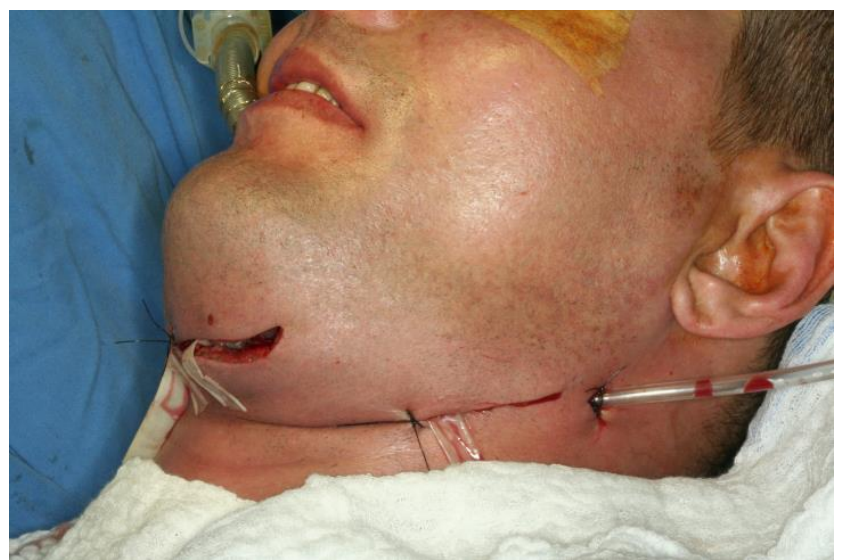

Figura 3: Aspecto logo após acessos cirúrgicos bilaterais e colocação de drenos.

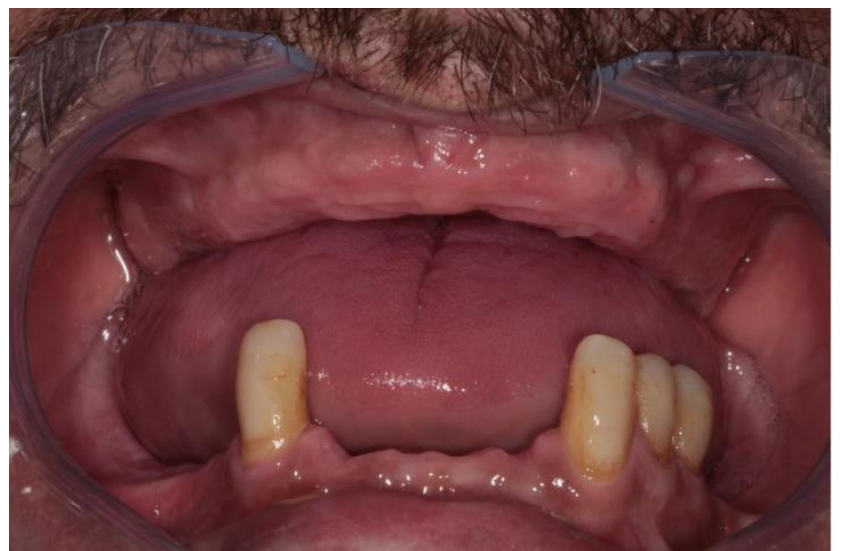

Figura 4: Condição bucal em que o paciente chegou à COU/UEL.
Foi então encaminhado para a Clínica Odontológica da UEL para reabilitação oral. Em sua consulta no Módulo de Clínica Integrada Protética, o paciente relatou ter sido internado devido a infecção odontogênica, o que gerou intenso trauma emocional. Foram realizadas consultas para instruções de higiene pois ainda se mostrava negligente em relação à saúde bucal. Diante das opções de tratamento, a reabilitação indicada para o caso e escolhida pelo paciente foi a confecção de prótese total superior e prótese parcial removível inferior (Figuras 5 e 6).

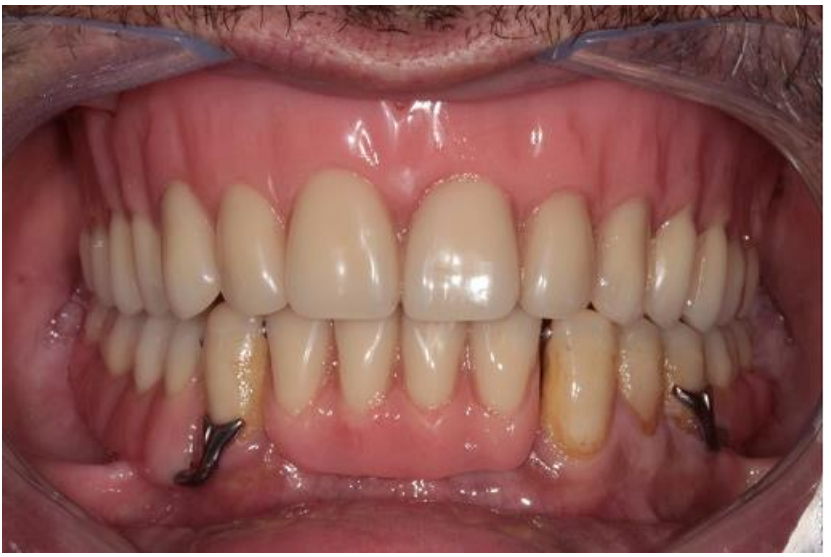

Figura 5: Paciente reabilitado com PT superior e PPR inferior

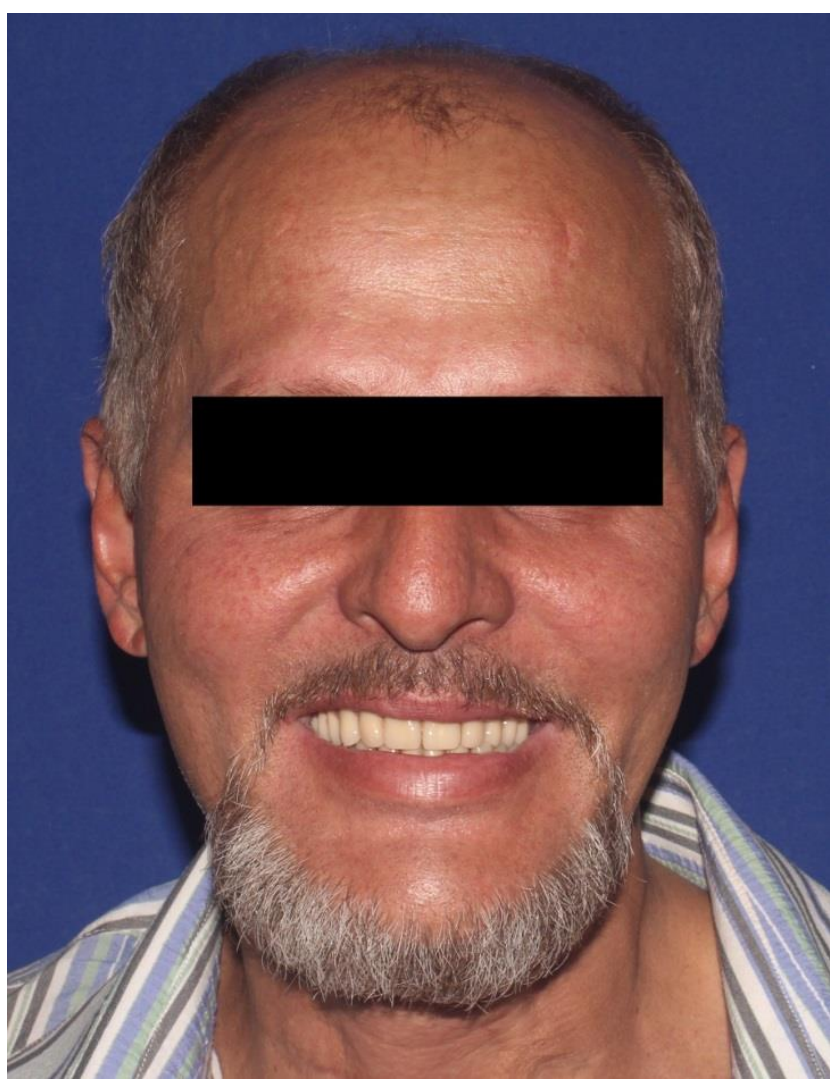

Figura 6: Aspecto facial do paciente após reabilitação.

\section{DISCUSSÃO}

A Angina de Ludwig é uma Infecção Cervical Profunda (ICP), ou seja, uma infecção caracterizada pela formação de celulite ou abscesso que acomete os planos fasciais profundos incluindo região cervical. Particularmente, a AL é uma doença 
infecciosa de progressão rápida que envolve os espaços submandibular, sublingual e submentoniano. Apesar da diminuição da incidência das ICP e das complicações associadas, elas ainda representam uma causa importante de morbidade e mortalidade, principalmente em pacientes imunocomprometidos e aqueles com baixo nível socioeconômico ${ }^{13}$.

Algumas condições como Diabetes Mellitus (DM), Hipertensão, infecção pelo HIV, além do tabagismo, etilismo e obesidade são comumente encontradas em pacientes com Angina de Ludwig, podendo ser consideradas como alguns dos fatores predisponentes para o desenvolvimento e agravamento das $\mathrm{ICPs}^{14,15}$. Sendo a DM a doença comórbida mais frequente nos casos de ICP, além de estar presente nos casos de maior gravidade ${ }^{16}$, o caso relatado não se apresenta em concordância neste aspecto, pois o quadro infeccioso evoluiu mal e foi de difícil estabilização mesmo o paciente não tendo nenhum comprometimento sistêmico. Porém, o paciente relatou ser etilista e tabagista que também são fatores predisponentes de AL.

Os microrganismos responsáveis por causarem as infecções de origem odontogênica, não raramente, apresentam resistência aos antibióticos empíricos. Estudos reportam de $14 \%$ até $81 \%$ de resistência bacteriana em casos de infecções maxilofaciais $^{17-19}$. A resistência bacteriana é um fator que colabora para a propagação e agravamento da infecção, e ocorre devido ao uso indiscriminado de antibióticos prescritos por dentistas ou usados sem prescrição médica/odontológica (automedicação). No presente caso, esta pode ser considerada uma das causas da grave evolução do quadro infeccioso, uma vez que o paciente relatou que havia episódios frequentes de dor mas que nunca procurava atendimento odontológico e se automedicava ${ }^{17}$. Deve-se suspeitar de resistência antibiótica em todos os pacientes que não respondem à abordagem cirúrgica inicial. A coleta para cultura microbiológica e teste de sensibilidade antimicrobiana são indicados em infecções severas, porém, a piora do quadro clínico é, frequentemente, notada antes mesmo de ter os resultados da cultura e de sensibilidade. Por isso, as decisões sobre a administração antimicrobiana são feitas, na maioria da vezes, com base em julgamento clínico $^{20}$. No caso relatado foi feita a coleta de material durante a abordagem cirúrgica pela equipe de CTBMF que teve resultado negativo na cultura microbiológica. Tal resultado pode ser explicado devido a coleta de material inapropriado ou até mesmo erro durante o processamento em laboratório. Assim sendo, a terapia antibiótica foi feita através da observação da evolução do quadro infeccioso, com auxílio e supervisão da equipe de Infectologia do hospital.

A AL pode causar obstrução e/ou infecção das vias aéreas, pneumonia, empiema pleural, mediastinite, pericardite, fasciíte necrosante cervicofacial, trombose da veia jugular, coagulação intravascular disseminada, trombose do seio dural e sepse, podendo levar o paciente a óbito. No caso relatado, o paciente desenvolveu um quadro de mediastinite, uma grave complicação da $\mathrm{AL}$, caracterizada pela infecção do espaço que acomoda os órgão vitais (pulmões e coração). Tung-Tsun Huang e colaboradores ${ }^{21}$, analisaram 185 casos de ICPs, nos quais 5 pacientes evoluíram para mediastinite e 2 destes morreram, o que nos confirma a gravidade dessa complicação associada à Angina de Ludwig.

O tempo de hospitalização depende da origem da infecção, doenças associadas, e da idade do paciente, podendo variar de 2 a 90 dias. Geralmente, os casos que levam mais tempo de internação hospitalar, são aqueles associados a Diabetes Mellitus ou pacientes imunosuprimidos ${ }^{22}$. Sakarya e colaboradores (2015), viram que a maioria dos pacientes acometidos por ICPs que participaram da pesquisa ficaram hospitalizados de 8 a 12 dias, e pacientes com complicações permaneceram a maioria de 15 a 31 dias $^{13}$. No presente caso, o paciente permaneceu em UTI por 10 dias e recebeu alta após 30 dias de internação hospitalar, o que é considerado um tempo estendido se considerarmos a não associação com condições sistêmicas.

Em relação à antibioticoterapia, no atendimento emergencial, o paciente foi tratado inicialmente com Penicilina Benzatina e Metronidazol. Devido à grave evolução do quadro infeccioso, a medicação foi trocada mais 3 vezes até estabilização. Em estudo recente, Varghese et al. ${ }^{23}$ concluíram que os antibióticos da classe das Penicilinas em associação a cobertura de gram negativos (Ex: Metronidazol) foi o suficiente para tratar a grande maioria dos casos de ICPs analisados no estudo. Apenas 7\% dos casos apresentou a necessidade de trocar a medicação com base no teste de sensibilidade aos antibióticos, casos que inclusive eram de pacientes diabéticos. Liau et al. ${ }^{24}$ também encontraram resultado semelhante analisando 672 pacientes; organismos resistentes a penicilina foram encontrados em $10,8 \%$, que necessitaram que uma maior estadia hospitalar e apresentaram maiores taxas de abordagem cirúrgica inicial não responsiva. É importante ressaltar que no tratamento da $\mathrm{AL}$ não é somente o antibiótico que contém a infecção, mas sim a combinação da drenagem cirúrgica, remoção da causa e administração farmacológica.

Como citado anteriormente, a remoção da causa da infecção é necessária, e muitas vezes as exodontias de um ou mais elementos dentários são inevitáveis. A gravidade do quadro infeccioso e as perdas dentárias são bastante traumáticas para o paciente no aspecto psicológico e social. A condição bucal influencia diretamente na qualidade de vida das 
pessoas tanto no nível biológico (mastigação, fonação e função articular) quanto nos níveis psicológico e social (autoestima, comunicação e estética) ${ }^{10}$. É por isso que o paciente deve ser motivado a mudar de atitude em relação à saúde oral a fim de prevenir outras complicações. Desta forma, a reabilitação pode ser indicada e contribuir para o aumento da autoestima e mudança de comportamento. Dentre as opções reabilitadoras, a Prótese Total (PT) e Prótese Parcial Removível (PPR) são capazes de devolver a autoestima ao paciente, restabelecendo função e estética dentro do padrão financeiro de pacientes de baixa renda. A condição financeira de cada paciente é um fator determinante para a escolha do tipo de reabilitação. No presente caso, houve indicação de instalação de PT superior e de PPR inferior e a decisão foi feita em conjunto com o paciente ${ }^{11,12}$. Com o incidente o paciente se conscientizou da necessidade do autocuidado, o qual foi reabordado por nós, destacando a importância da manutenção da saúde bucal para evitar novos episódios de infecção.

\section{CONCLUSÃO}

$\mathrm{O}$ caso abordado nos mostra que mesmo com o avanço em relação a saúde bucal, ainda existem casos de infecção odontogênica negligenciados que podem agravar e colocar a vida do paciente em risco. Desta forma, a Angina de Ludwig sendo uma condição importante deve ser tratada de forma emergencial para evitar maiores complicações. A reabilitação do paciente com histórico de infecção é indicada para restabelecer a função, estética e principalmente qualidade de vida a partir da condição bucal saúdavel.

\section{REFERÊNCIAS}

1. Jiménez Y, Bagán JV, Murillo J, Poveda R. Odontogenic infections. Complications. Systemic manifestations. Med Oral Patol Oral Cir Bucal. 2004;9(Suppl):139-43.

2. Lugo AFG, Ravago MGC, Martinez RAG, Peltrini RJZ. Ludwig's angina: A report of two cases. Rev Esp Cir Oral Maxilofac. 2014;36(4):177-81.

3. Umeda M, Minamikawa $T$, Komatsubara $H$, Shibuya Y, Yokoo S, Komori T. Necrotizing fasciitis caused by dental infection: a retrospective analysis of 9 cases and a review of the literature. Oral Surg Oral Med Oral Pathol Oral Radiol Endod. 2003;95(3):283-90.

4. Bakir S, Tanriverdi MH, Gün R, Yorgancilar AE, Yildirim M, Tekbas $G$ et al. Deep neck space infections: a retrospective review of 173 cases. Am J Otolaryngol. 2012;33(1):56-63.

5. Brook I. Microbiology and management of peritonsillar, retropharyngeal, and parapharyngeal abscesses. J Oral Maxillofac Surg. 2004;62(12):1545-50.
6. Caccamese JF Jr, Coletti DP. Deep neck infections: Clinical considerations in aggressive disease. Oral Maxillofac Surg Clin North Am. 2008;20(3):367-80.

7. Chen MK, Wen YS, Chang CC, Lee HS, Huang MT, Hsiao HC. Deep neck infections in diabetic patients. Am J Otolaryngol. 2000;21(3):169-73

8. Vieira F, Allen SM, Stocks RM, Thompson JW. Deep neck infection. Otolaryngol Clin North Am. 2008;41(3):459-83.

9. Zarb GA, Bolender CL. Tratamento protético para os pacientes edêntulos - Próteses totais convencionais e implantossuportadas. 13. ed. Santos: São Paulo; 2013.

10.Rodrigues JC. Tabu do corpo. Rio de Janeiro: Fiocruz; 2006.

11.Narby B, Kronström M, Söderfeldt B, Palmqvist S. Changes in attitudes toward desire for implant treatment: a longitudinal study of a middle-aged and older Swedish population. Int J Prosthodont. 2008;21(6):481-85.

12.Walton JN, MacEntee MI. Choosing or refusing oral implants: a prospective study of edentulous volunteers for a clinical trial. Int $\mathbf{J}$ Prosthodont. 2005;18(6):483-8.

13.Sakarya EU, Kulduk E, Gündoğan O, Soy FK, Dündar R, Kilavuz AE, Özbay C, Eren E, İmre A. Clinical features of deep neck infection: analysis of 77 patients. Kulak Burun Bogaz Ihtis Derg. 2015;25(2):102-8.

14.Botha A, Jacobs F, Postma C. Retrospective analysis of etiology and comorbid diseases associated with Ludwig's Angina. Ann Maxillofac Surg. 2015;5(2):168-73.

15. Suehara AB, Goncalves AJ, Alcadipani FAMC, Kawabata NK, Menezes MB. Deep neck infection: analysis of 80 cases. Braz J Otorhinolaryngol. 2008;74(2):253-59.

16.Lee JK, Kim HD, Lim SC. Predisposing factors of complicated deep neck infection: an analysis of 158 cases. Yonsei Med J. 2007;48(1):55-62.

17.Igoumenakis D, Gkinis G, Kostakis G, Mezitis M, Rallis G. Severe odontogenic infections: causes of spread and their management. Surg Infect (Larchmt). 2014;15(1):64-8.

18. Rao DD, Desai A, Kulkarni RD, Gopalkrishnan $\mathrm{K}$, Rao CB. Comparison of maxillofacial space infection in diabetic and nondiabetic patients. Oral Surg Oral Med Oral Pathol Oral Radiol Endod. 2010;110(4):e7-12.

19.Akinbami BO, Akadiri O, Gbujie DC. Spread of odontogenic ifnfections in Port Harcourt, Nigeria. J Oral Maxillofac Surg. 2010;68(1):2472-77.

20.Flynn TR, Shanti RM, Hayes C. Severe odontogenic infections, Part 2: Prospective outcomes study. J Oral Maxillofac Surg. 2006;64(7):1104-13. 
21.Huang TT, Liu TC, Chen PR, Tseng FY, Yeh TH, Chen YS. Deep neck infection: analysis of 185 cases. Head Neck. 2004;26(10):854-60.

22.Hsu RF, Wu PY, Ho CK. Transcervical drainage for descending necrotizing mediastinitis may be sufficient. Otolaryngol Head Neck Surg. 2011;145(5):742-47.

23. Varghese L, Mathews SS, Antony Jude Prakash J, Rupa V. Deep head and neck infections: outcome following empirical therapy with early generation antibiotics. Trop Doct. 2018;48(3):179-82.

24.Liau I, Han J, Bayetto K, May B, Goss A, Sambrook $\mathrm{P}$ et al. Antibiotic resistance in severe odontogenic infections of the South Australian population - a 9- year retrospective audit. Aust Dent J. 2018;63(2):187-92.

\section{CONFLITO DE INTERESSES}

Os autores declaram não haver conflitos de interesse.

\section{AUTOR PARA CORRESPONDENCIA}

\section{Cecília Luiz Pereira Stabile}

ceciliastabile@gmail.com

Submetido em 28/10/2018

Aceito em 12/03/2019 